\title{
Artificial Intelligence Techniques Evaluation for Modulation Format Identification in Optical Networks
}

\author{
André Luiz Nunes de Souza(D), Tiago Sutili(i), \\ José Hélio da Cruz Júnior $\mathbb{B}$, and Rafael Carvalho Figueiredo \\ Optical Communication Solutions, CPQD, Campinas-SP, Brazil \\ aluizs@cpqd.com.br, tsutili@cpqd.com.br,hcruz@cpqd.com.br,and rafaelcf@cpqd.com.br
}

\begin{abstract}
In an elastic network paradigm, where the transceiver is able to control several characteristics of the transmitted signal according to the optical link quality and capacity demand, receivers able to automatically detect the modulation format are fundamental to recover the transmitted signal without the necessity of headers that reduce system capacity. This work presents a simulated performance comparison of six methods for blind identification of modulation format in high-capacity optical systems: k-nearest neighbors (KNN), k-means, fuzzy c-means, deep neural networks, support-vector machine (SVM) and peak-to-average power ratio (PAPR). The transmitted channels were 64-GBd modulated with the following modulation formats available at the transceiver: QPSK, 16QAM, 64QAM, and 256QAM. The optical link was emulated considering several impairments, as amplified spontaneous emission from optical amplifiers, frequency and phase noise from lasers, and polarization rotation and differential group delay from the propagation. The support-vector machine algorithm presented the most robust results.
\end{abstract}

Index Terms - digital signal processing, elastic optical networks, modulation format blind identification.

\section{INTRODUCTION}

Optical networks are a key technology to support the global telecommunications backbone, especially with the rising and large acceptance of on-demand high-quality video distribution, cloud computing, and Internet of Things (IoT) applications. This new technological paradigm, besides increasing the capacity demand in optical networks, drastically changes its requirements in some cases. Depending on the application, there are different specifications in terms of throughput, latency, energy consumption, and/or reach [1]. In specific, high-capacity optical systems usually rely on wavelength-multiplexing (WDM) techniques, on which the specific frequency (or wavelength) of each transmitted channel is set in a fixed grid. As defined by the International Telecommunication Union - Telecommunication Standardization Sector (ITU-T recommendation G.694.1 [2]), one of the most usual channel grid defines a 50-GHz spacing between channels, implicating that, fundamentally, each channel will occupy a 50-GHz fixed slot, despite its actual spectral width. In that way, optical components and devices can be designed to operate in specific and fixed frequency slots, diminishing costs and accelerating the development process.

To support the increasingly higher and more heterogeneous demand for transmission capacity and to improve optical systems spectral efficiency, the concept of elastic optical networks (EON) was intro- 
duced in 2012 [3]. In this new paradigm, the optical transceivers must be able to adapt their transmission rate and modulation format, among other operational parameters, based on the optical channel quality of transmission (QoT) and bit rate demand independently for each wavelength-multiplexed channel. Several previous works in the state-of-the-art already demonstrated the advantages of elastic optical networks, especially regarding the capital (CAPEX) and operational expenditures (OPEX) and the network maximum transmission capacity [4], [5]. In this scenario, optical receivers with the capacity to automatically identify the transmitted modulation format are a key technology to correctly implement the digital signal processing (DSP) stack and, ultimately, to correctly decode the transmitted bit sequence without additional headers, increasing the systemic net transmission capacity in an EON-based optical network.

In this context, the recent development of machine learning (ML) based techniques in computer vision and classification problems inspired its application in several areas, among them the blind modulation format identification (MFI) problem specifically in high capacity optical networks. For that, several machine learning based algorithms have been proposed to be applied directly on the received optical constellation. In [6], the authors describe a modulation format identification method based on the $k$ nearest neighbors algorithm, employing as inputs the histograms of the real and imaginary components of the received optical signal sampled at one sample per symbol. In [7], the k-means is demonstrated as a valid MFI method in optical communications, allowing the received constellation clustering, which, based on the quantity and position of clusters, allows the modulation format identification. In [8], the authors demonstrate that deep neural networks (DNN) are capable to effectively identify the transmitted modulation format for different optical signal-to-noise ratios (OSNR) even in the presence of other degrading phenomena, as frequency and phase deviations and nonlinear Kerr-related effects. Alternatively, analytical methods can also be employed to blindly identify the transmitted modulation format. Among them, some works had proposed to employ the received signal entropy [9] or peak-toaverage power ratio (PAPR) [10] to extract data about the transmitted optical constellation.

Considering the several different modulation format identification approaches proposed in the literature, this work aims to directly compare different supervised and unsupervised machine learning proposals with an analytical method as a performance reference. For that, in order to assess each implementation performance in different scenarios, a simulation setup was employed, allowing to directly compare each implementation accuracy, depending on the degrading imposed by different impairments. In the proposed simulation, 64-GBd optical channels are transmitted with constellations following the QPSK, 16QAM, 64QAM, and 256QAM modulation formats. To assess each considered method's resilience to different transmission effects, the simulation setup includes degrading sources as additive white Gaussian noise (AWGN), phase noise, residual frequency deviation, polarization rotation, and differential group delay. Based solely on the data acquired by a coherent receiver, algorithms based on deep neural networks (DNN), support-vector machine (SVM), k-nearest neighbors, k-means, and fuzzy c-means are employed to identify the transmitted modulation format. On one hand, the DNN, the k-nearest neighbors, and the SVM algorithms are employed to identify and classify patterns on the received data. On the other, the clustering methods (e.g., k-means and fuzzy c-means), allow one to find the optimum number of clusters on the received data. As a comparison metric, the analytical method based on peak-to-average power ratio (PAPR) is also considered.

This work aims to offer a comprehensive performance comparison between several artificial intelli-

Brazilian Microwave and Optoelectronics Society-SBMO Brazilian Society of Electromagnetism-SBMag received 29 Jan 2021; for review 29 Jan 2021; accepted 29 Apr 2021 (c) 2021 SBMO/SBMag (cc) BY ISSN 2179-1074 
gence based modulation format identification techniques in the context of the next-generation optical communication systems. All the investigated techniques were previously proposed in the technical literature for this specific application or classification problems in general. However, they had to be adapted and optimized to maximize their accuracy considering modulation formats up to 256 symbols with 64-GBd symbol rate. Furthermore, the proposed simulation-based investigation allows one to assess the individual accuracy penalty due to several optical transmission impairments for each technique. The present work is an extension of the results presented in [11], including the performance analysis of two more MFI algorithms (fuzzy c-means and SVM) and the dynamic equalizer optimization (in terms of its update step, number of filter taps, and the duration of the convergence) to ensure a better compensation of polarization rotation and differential group delay. It is divided as follows: in Section II the methods' implementations are reviewed with the definition of its hyperparameters; in Section III the simulation setup is described with the definition of each transmission scenario employed in the comparative analysis; in Section IV the obtained results are discussed; finally, in Section V the work conclusions are presented, allowing the definition of future works related with the carried out investigation.

\section{Methodologi}

The proposed digital signal processing (DSP) stack for coherent optical transceivers with modulation format identification is represented in Fig. 1, encompassing the following blocks: (1) channel conditioning; (2) chromatic dispersion (CD) compensation; (3) timing error correction; (4) optical carrier frequency deviation compensation; (5) channel dynamic equalization; (6) optical carrier phase deviation recovery. As the operation of many of these blocks are independent from the transmitted modulation format (as the case for blocks 1,2,3, and 4), they can be executed before the modulation format identification algorithm, simplifying the MFI implementation and increasing its performance. Therefore, as highlighted in Fig. 1, the MFI algorithms must be placed between blocks 4 and 5 . As previously discussed, the performance of MFI approaches based on k-nearest neighbors (KNN), $k$-means, fuzzy c-means, deep neural networks, support-vector machine (SVM), and peak-to-average power ratio (PAPR) algorithms will be compared. All ML-based methods were implemented in Python employing the scikit-learn (KNN, k-means, DNN and SVM) and skfuzzy (fuzzy c-means) toolboxes. The configuration parameters and kernel functions were selected through cross-validation. Each hyperparameter was optimized separately. Starting at the default value, the value was increased/decreased until reaching a maximum identification accuracy at the training data. If more than one hyper-parameter had to be selected for a function, the search for the maximum was done by successive approximations (i.e., each parameter was optimized at a time) considering the optimal value found in previous iterations for the other parameters. This iterative process was repeated until convergence of the optimal values.

Specifically for the k-nearest neighbors, as proposed in [6], 10000 signal samples after decimation are considered to compose amplitude histograms (AH) for its real and imaginary components. The frequency value for each histogram interval is an input parameter to the model. To reduce its computational complexity and improve the algorithm resilience, the authors propose to slice the signal real and imaginary components in 100 intervals from -1.5 up to +1.5 and defining $F_{i}$ as the frequency for each interval $i$. As proposed by the authors, the parameter effectively sent to the model will be: 0 if $F_{i}<32 ; 32$ if $F_{i}>256$; and $F_{i} / 8$ for the other cases. Finally, the model considers a uniform weight equal to 5 for the 6 neighbors with shortest Euclidean distances. 


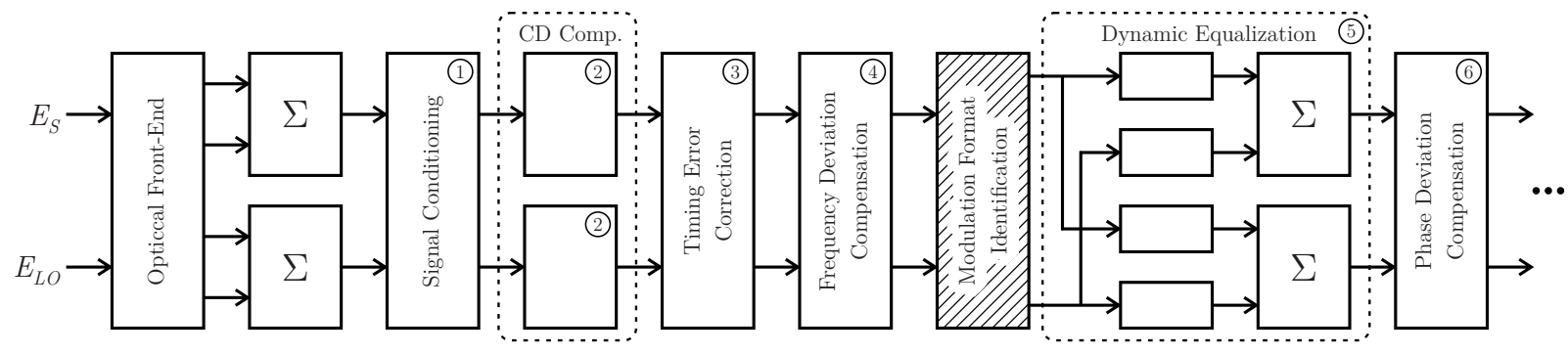

Fig. 1. Block diagram representing a conventional coherent receiver with the main DSP stack blocks. In the figure, $E_{S}$ and $E_{L O}$ stands for the received signal and local oscillator electromagnetic fields, respectively.

Following, for the k-means implementation considering one sample per received symbol, as proposed in [7], the authors implemented a pre-processing stage for both $x$ and $y$ optical polarizations, mapping them into the Stokes space as follows:

$$
S=\left(\begin{array}{c}
S_{0} \\
S_{1} \\
S_{2} \\
S_{3}
\end{array}\right)=\frac{1}{2}\left(\begin{array}{c}
|x|^{2}+|y|^{2} \\
|x|^{2}-|y|^{2} \\
2 \cdot \operatorname{real}(x \bar{y}) \\
2 \cdot \operatorname{imag}(x \bar{y})
\end{array}\right) .
$$

The clustering is carried out in the three-dimensional space defined by vectors $S_{1}, S_{2}$, and $S_{3}$ normalized by $S_{0}$. As a consequence, the QPSK modulation format will be defined by 4 clusters in the Stokes space, the $16 \mathrm{QAM}$ by 60 , the $64 \mathrm{QAM}$ by 972 , and the $256 \mathrm{QAM}$ by 15720 . However, with this approach, the number of clusters in the Stokes space for the higher-order modulation formats is impractical, demanding a large number of samples to be processed and, as a consequence, an undesirable increase in system latency. Alternatively, in this work, the clustering is carried out based solely on the received signal, reducing the number of clusters to the number of constellation points. The model was implemented following the Elkan algorithm [12] with aleatory initial centroid positions and a maximum of 300 iterations. The Silhouette score [13] was employed to select the optimum number of clusters for each signal following the description of [14].

The clustering based on the fuzzy c-means method was also performed directly on the received signal and the Silhouette coefficient was used to select the optimum number of clusters, following the proposed in [14]. The method was implemented as proposed in [15] with aleatory initial centroid positions and a maximum of 100 iterations. The $k$-means and fuzzy c-means have the advantage of not requiring any prior training as they are examples of 'lazy-training' methods. However, for this reason, they require more online calculations and, therefore, a higher latency.

For the MFI based on deep neural networks, as proposed in [8], amplitude histograms (AH) obtained from the absolute values of each transmitted symbol are divided in 80 intervals, which frequencies are the inputs for a deep neural network with two hidden layers. These layers are composed of 30 and 10 neurons, respectively, and operate as autoencoders with a rectified linear activation function (ReLU). Following, the output layer is a perceptron layer with softmax activation function. The ADAM optimization algorithm was employed with a constant learning rate equal to 0.001 and minibatch size equal to 200 .

Finally, the support-vector machine was implemented using the SVC classifier of the scikit-learn Python toolbox. The kernel function used was the $r d f$ with parameters $\gamma$ and $c$ equal to 0.0001 and 
1, respectively. Its inputs are the same amplitude histograms used by the DNN and KNN methods but with its frequencies normalized, since the SVM method is highly scale dependent.

As a comparative reference, the peak-to-average power ratio was also employed as an analytical method to identify the transmitted modulation formats. One major limitation from this approach is related to the fact that the PAPR depends on the level of noise present in the received signal and, as a consequence, the decision thresholds must consider the received signal optical signal-to-noise ratio (OSNR). In this work, the decision thresholds were selected as the intermediate point between the PAPR of each modulation format, as presented in [10]. There are several proposals in the literature to estimate the received signal OSNR [16], however, for simplicity, in this work, it is assumed that the OSNR value is always fully known. Even though the PAPR solution is the most computationally simple, the additional complexity of the OSNR estimator must be considered for the selection of the best MFI solution.

\section{Simulation Setup}

The optical system simulation is based in a back-to-back link with amplified spontaneous emission (ASE) loading to emulate the noise from optical amplifiers and to allow the ranging on the received optical signal-to-noise ratio (OSNR), as depicted in Fig. 2. Besides the setup simplicity, it is suitable to emulate signal degradation due to effects whose compensation requires algorithms that are affected by the transmitted modulation format.

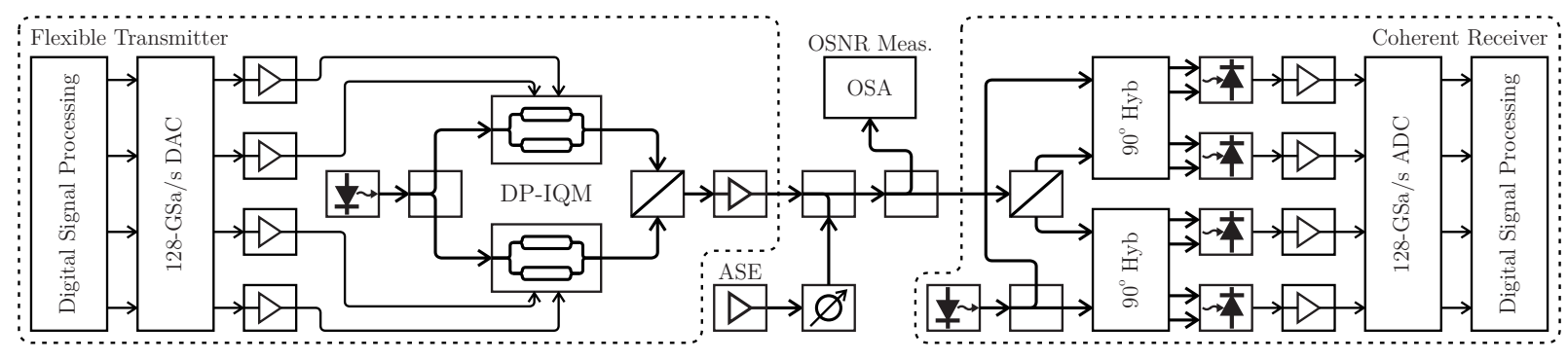

Fig. 2. Simulation setup employing an amplified spontaneous emission (ASE) noise loading method to range the received optical signal-to-noise ratio (OSNR).

At the transmitter, a pseudorandom binary sequence (PRBS) composed of $2^{18}$ bits is independently generated to compose the data to be transmitted through each multiplexed optical polarization. Following, the bit sequence is mapped into the selected modulation format, which, as previously discussed, will be randomly chosen among QPSK, 16QAM, 64QAM, and 256QAM. The resulting symbols are oversampled to 2 samples per symbol and filtered by a raised cosine shaping filter with a roll-off factor equal to 0.1 . The four modulating signals are converted to the analog domain by a digital-to-analog converter (DAC) with sampling rate equal to $128 \mathrm{GSa} / \mathrm{s}, 3-\mathrm{dB}$ electrical bandwidth equal to $36 \mathrm{GHz}$, and 8-bits nominal resolution. The analog signals are linearly amplified by microwave drivers and applied in the arms of a dual-polarization in-phase and quadrature optical modulator (DP-IQM). The modulated optical carrier is generated by an external cavity laser (ECL) with its linewidth ranged to assess the phase noise impact on the MFI algorithms performance.

Furthermore, to emulate the transmitted signal polarization state deviation, the polarization-multiplexed optical modulated signal is multiplied with the Jones matrix: 


$$
J=\left(\begin{array}{cc}
\cos \theta \cdot e^{-j \tau / 2} & -\operatorname{sen} \theta \cdot e^{-j \tau / 2} \\
\operatorname{sen} \theta \cdot e^{j \tau / 2} & \cos \theta \cdot e^{j \tau / 2}
\end{array}\right)
$$

on which $\tau$ represents the differential group delay (DGD) and $\theta$ the polarization rotation angle. Next, to emulate the amplifiers' noise in a conventional optical link, amplified spontaneous emission is loaded into the transmitted signal to achieve the desired optical signal-to-noise ratio at the optical front-end.

The optical coherent receiver with polarization diversity has a $36-\mathrm{GHz}$ bandwidth and is composed of the combination of polarization beam splitters (PBS), optical couplers, $90^{\circ}$ optical hybrids, a second external cavity laser as the local oscillator, and a set of balanced photodiodes with transimpedance amplifiers. The signals converted to the electrical domain are sampled by a 128-GSa/s analog-to-digital converter (ADC) with 8-bits nominal resolution and $36-\mathrm{GHz} 3-\mathrm{dB}$ bandwidth. The resulting digital signals are acquired to be post-processed by the DSP stack depicted in Fig. 1, employing each one of the proposed MFI algorithms. The analyzed dataset is composed of 600 sequences for each modulation format and received OSNR, each one being composed of 10000 symbols. To avoid the necessity of knowing the received OSNR, all methods that require training were trained using $1 / 3$ of the dataset sequences at OSNR $=26.4 \mathrm{~dB}$ (i.e., 200 sequences for each modulation format, 800 sequences in total). As a consequence, the MFI performance analysis was performed using $2 / 3$ of the generated sequences for all OSNR values (i.e., 400 sequences for each modulation format, 1600 sequences in total for each OSNR point). The figure of merit selected to compare the performance of the MFI approaches is the accuracy as a function of the OSNR. The accuracy for multi-class classification is calculated as the average accuracy of the classifiers for each class, as given in [17]. The properties of this figure of merit in terms of robustness to present an algorithm's overall performance, without being affected by the performance on a specific class, is well suited to the problem here analyzed, as the interest is to evaluate if the algorithm is able to correctly identify the modulation format during the whole operation of the device. The analysis is complemented by confusion matrices to get insights into the performance of the per-class classification. The required OSNR value to achieve $100 \%$ may be used to predict the maximum transmission distance based on perturbative models. The relation between required OSNR and transparent transmission distance may be calculated using the GN model for C-band transmissions, as have been done in [18], or, for multi-band scenarios, the Generalized GN model (GGN) [19] or the ISRS GN model [20].

\section{RESULTS}

The accuracy for each MFI approach as a function of the received signal OSNR is presented in Fig. 3, on which the signals were degraded only by the insertion of ASE emulated by loading AWG noise (i.e., in this particular case, the signals were not affected by phase, frequency, or polarization deleterious effects). The dataset sequences acquired specifically to train the machine learning approaches were also degraded only by the insertion of AWG noise with an OSNR equal to $26.4 \mathrm{~dB}$. The presented results indicate that the KNN, $k$-means, SVM, and PAPR algorithms have a similar performance in this condition, achieving a 100\% accuracy for OSNRs higher than $20.8 \mathrm{~dB}$. Nevertheless, the deep neural network required an OSNR $2 \mathrm{~dB}$ higher to attain $100 \%$ accuracy, while the fuzzy c-means was not able to correctly identify the 256QAM modulation format, probably due to the great number of clusters.

Brazilian Microwave and Optoelectronics Society-SBMO Brazilian Society of Electromagnetism-SBMag received 29 Jan 2021; for review 29 Jan 2021; accepted 29 Apr 2021 (c) 2021 SBMO/SBMag (cc) BY

ISSN 2179-1074 


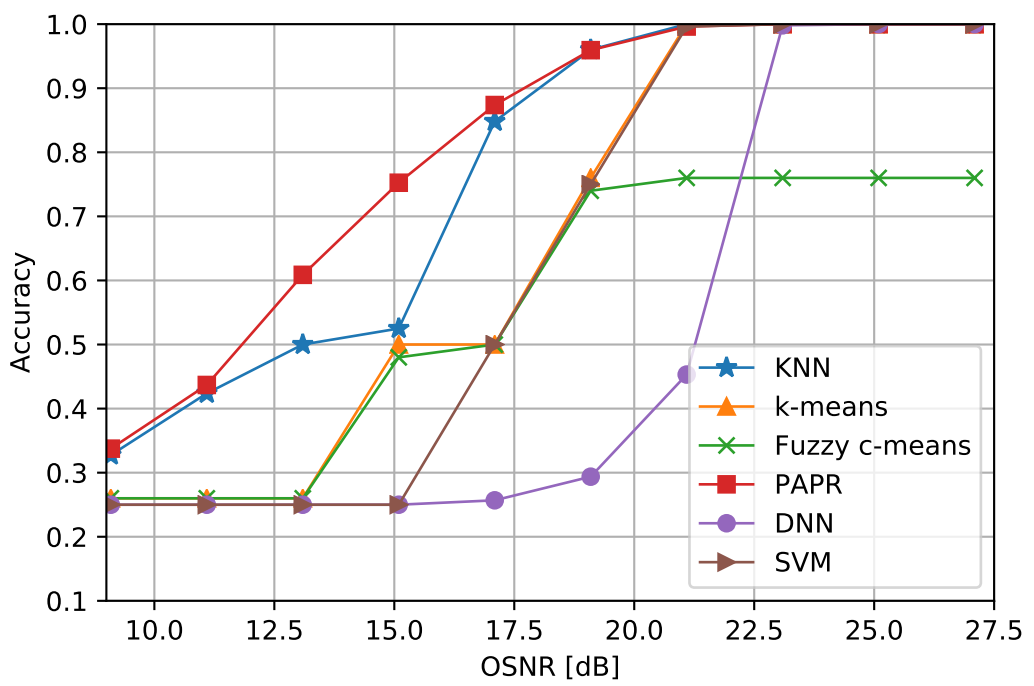

Fig. 3. Average accuracy of the MFI methods as a function of the received OSNR for the ASE-only scenario.

Following, the results presented in Fig. 4 consider also signal degradation due to lasers with uniformly distributed residual frequency deviation and linewidth between 0 and $1 \mathrm{MHz}$ and between 0 and $100 \mathrm{kHz}$, respectively. The frequency and phase distortions will induce undesirable rotations on the received signal, misshaping the Gaussian shape of each symbol and, as a consequence, spreading the acquired constellation points. Due to the nature of these distortions, the MFI approaches that are based solely on the analysis of data obtained from the signals amplitude information (i.e., as the DNN, SVM, and PAPR) are not affected by phase and frequency distortions and are not considered at this moment. Thus, the results only present the comparative analysis between the k-means, fuzzy c-means, and KNN algorithms, in all cases presenting the previously obtained results considering only ASE degradation as a reference. Furthermore, the training sequences were obtained from signals with 26.4-dB OSNR and with phase and frequency deviations ranging uniformly from 0 up to the maximum values considered on the analysis presented in Fig 4 (i.e., 1-MHz residual frequency deviation and 100-kHz lasers linewidth).

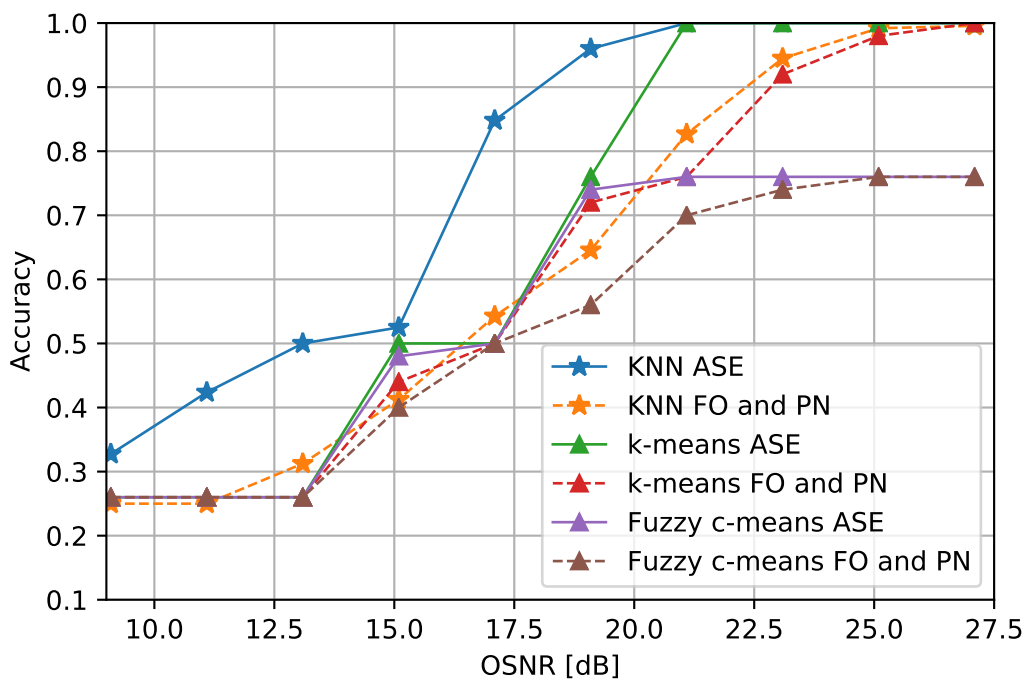

Fig. 4. Average accuracy of the KNN, k-means, and fuzzy c-means methods as a function of the received OSNR for the scenario with ASE and with frequency offset (FO) and phase noise (PN).

Brazilian Microwave and Optoelectronics Society-SBMO Brazilian Society of Electromagnetism-SBMag received 29 Jan 2021; for review 29 Jan 2021; accepted 29 Apr 2021 (C) 2021 SBMO/SBMag (cc) BY

ISSN 2179-1074 
For both the KNN and $k$-means methods, it is shown a 5 -dB penalty in the OSNR required to achieve a $100 \%$ accuracy in comparison with the scenario in which the signals were only degraded by ASE insertion. Meanwhile, the fuzzy c-means shows a penalty of $4 \mathrm{~dB}$ to attain its best performance, but it never reaches $100 \%$ accuracy in the range of OSNRs of interest, similarly to the ASE-only analysis. A possible solution to improve the performance of the methods in this scenario could be the creation of k-means, fuzzy c-means, and KNN models based only on the amplitude histogram, ignoring the phase and frequency data as is the case for the DNN, SVM and PAPR approaches. However, it would make it impossible to differentiate modulation formats based solely on phase-keying techniques, such as QPSK, 8PSK, 16PSK, among others.

Lastly, the MFI algorithms performance in a system impacted also by a maximum of half-symbol DGD and $45^{\circ}$ polarization rotation is presented in Fig. 5 for the k-means and fuzzy c-means algorithms, in Fig. 6 for the PAPR and DNN algorithms, and in Fig. 7 for the SVM and KNN algorithms. The presented results also compare the impact of the constant modulus algorithm (CMA) [21] in the MFI performance since it is commonly employed as a pre-convergence stage to adapt the dynamic equalization filters. However, it is well reported in the literature that it presents a poor performance when applied in systems based on high-order modulation formats since it rarely converges to its optimum solution at this condition. Despite this limitation, as it does not require previous knowledge of the transmitted modulation format, it can be employed in a stage allowing the DSP stack to separate the transmitted signal optical polarization components before the MFI algorithm. In this case, the training dataset was acquired considering signals degraded by AWG noise with an OSNR equal to $26.4 \mathrm{~dB}$, phase and frequency deviations ranging, uniformly, from 0 up to the maximum values employed in the analyzed signals (i.e., 1-MHz residual frequency deviation and 100-kHz lasers linewidth), and DGD and polarization rotation ranging from 0 up the values employed in the analyzed signals (i.e., half-symbol and $45^{\circ}$, respectively). In the cases employing the CMA for pre-equalization, 30000 extra samples were generated to be used to pre-converge the equalization algorithm with 12-tap filters and an update step $\mu$ equal to 0.001 .

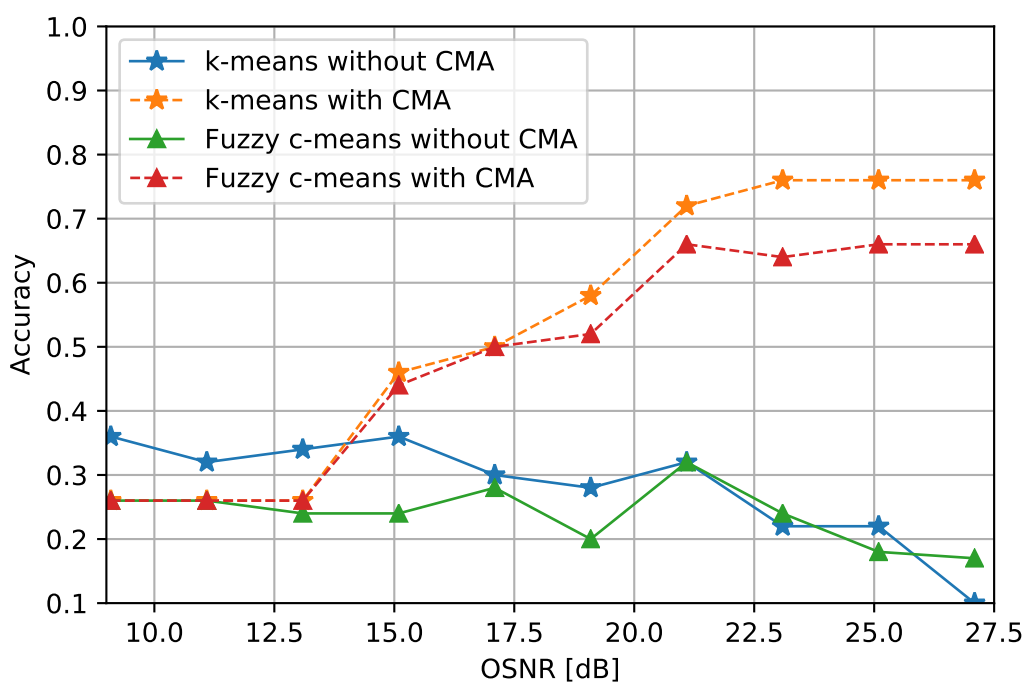

Fig. 5. Average accuracy of the k-means and fuzzy c-means methods, with and without pre-equalization using the CMA algorithm, as a function of the received OSNR for the scenario considering ASE noise, phase noise, residual frequency offset, DGD, and polarization rotation.

Brazilian Microwave and Optoelectronics Society-SBMO Brazilian Society of Electromagnetism-SBMag received 29 Jan 2021; for review 29 Jan 2021; accepted 29 Apr 2021 (c) 2021 SBMO/SBMag (cc) BY

ISSN 2179-1074 
The results indicate that all algorithms were severely affected by the DGD and polarization rotation effects, being unable to achieve accuracy close to $100 \%$ when the CMA pre-equalization is not performed. Specifically for the k-means and fuzzy c-means, it is also possible to notice that the CMA was not able to sufficiently compensate the DGD and polarization rotation degradation effects, degrading the overall accuracy of both algorithms. The PAPR and KNN algorithms were also impacted by the equalization errors and did not achieve $100 \%$ accuracy in the range of OSNRs of interest.

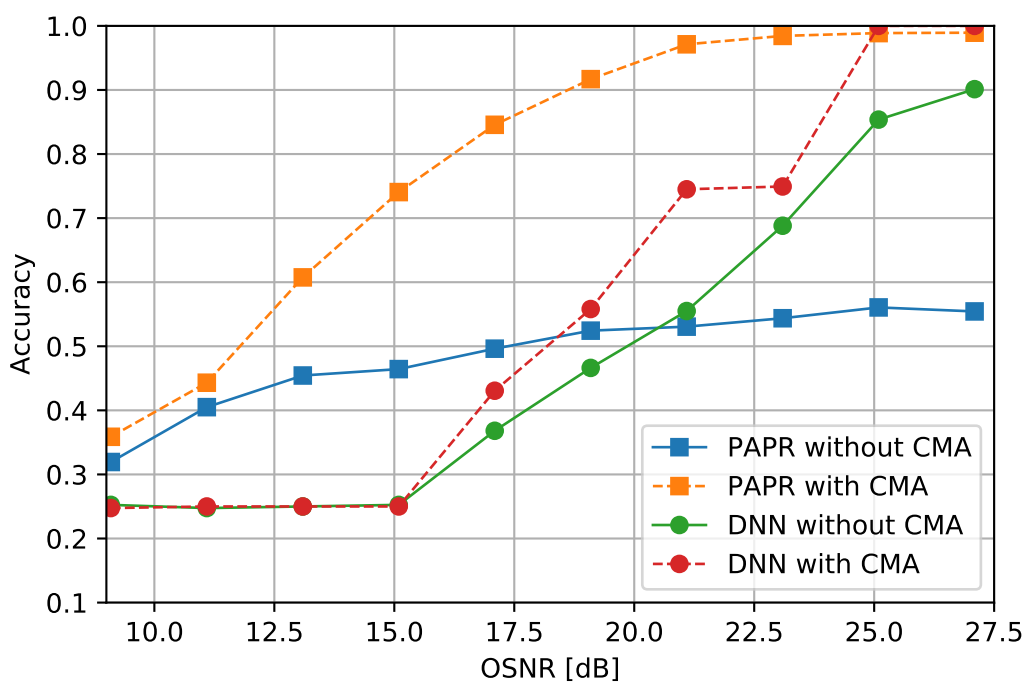

Fig. 6. Average accuracy of the PAPR and DNN methods, with and without pre-equalization using the CMA algorithm, as a function of the received OSNR for the scenario considering ASE noise, phase noise, residual frequency offset, DGD, and polarization rotation.

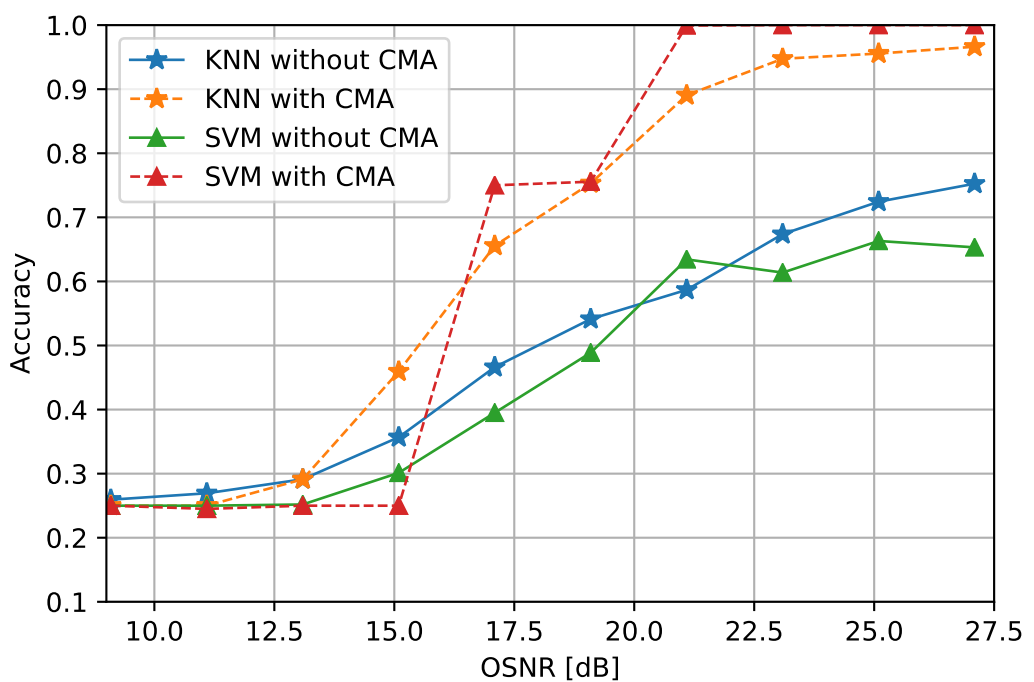

Fig. 7. Average accuracy of KNN and SVM methods, with and without pre-equalization using the CMA algorithm, as a function of the received OSNR for the scenario considering ASE noise, phase noise, residual frequency offset, DGD, and polarization rotation.

The deep neural network showed a penalty to the ASE-only scenario of $2.5 \mathrm{~dB}$. Surprisingly, the performance of the support-vector machine method does not seem to be impacted by the imperfect equalization of polarization rotation and DGD. It achieved 100\% accuracy at the same received OSNR 
as in the ASE-only scenario (i.e., $20.8 \mathrm{~dB}$ ). Even though the required OSNR values were the same, to claim that there was no penalty would require the simulation of many intermediate OSNR points. With the present results, we can only state that the penalty suffered by the SVM in relation to the ASE-only case was less than $2 \mathrm{~dB}$. The results for an OSNR equal to $27.1 \mathrm{~dB}$ are summarized in the confusion matrices presented in Fig. 8 .

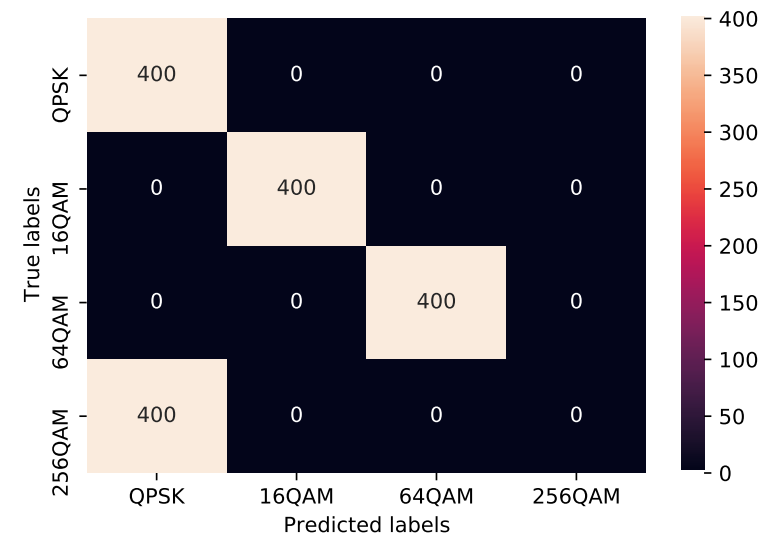

(a)

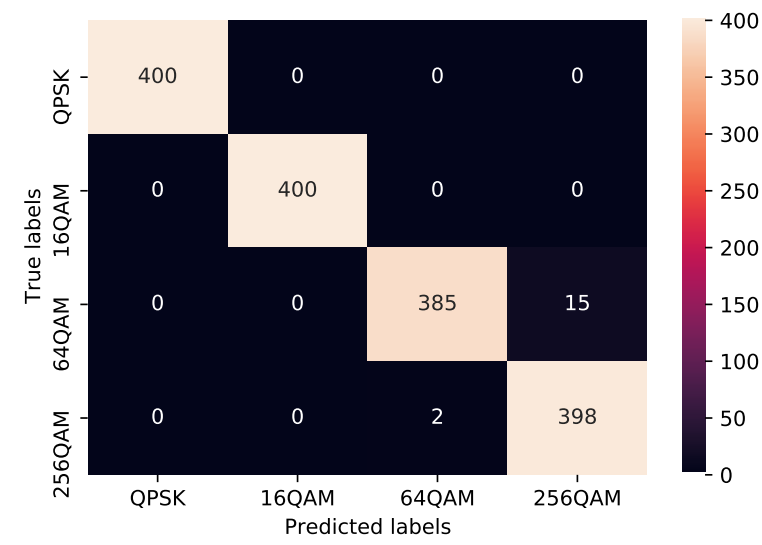

(c)

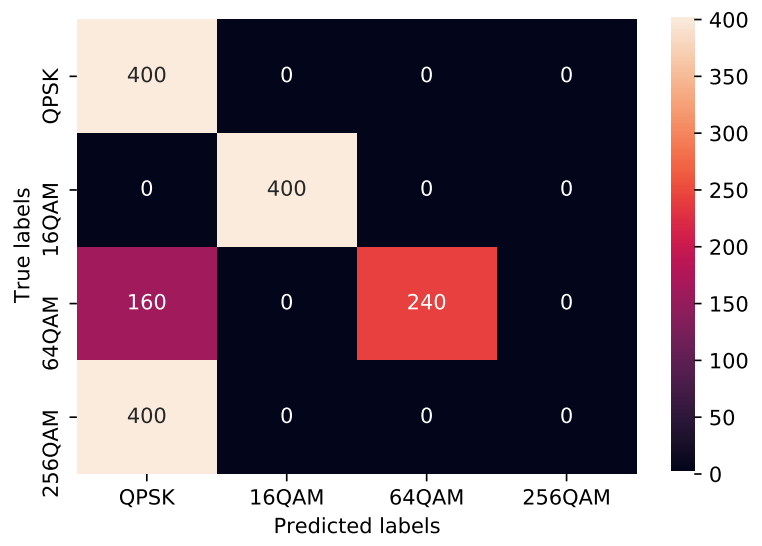

(b)

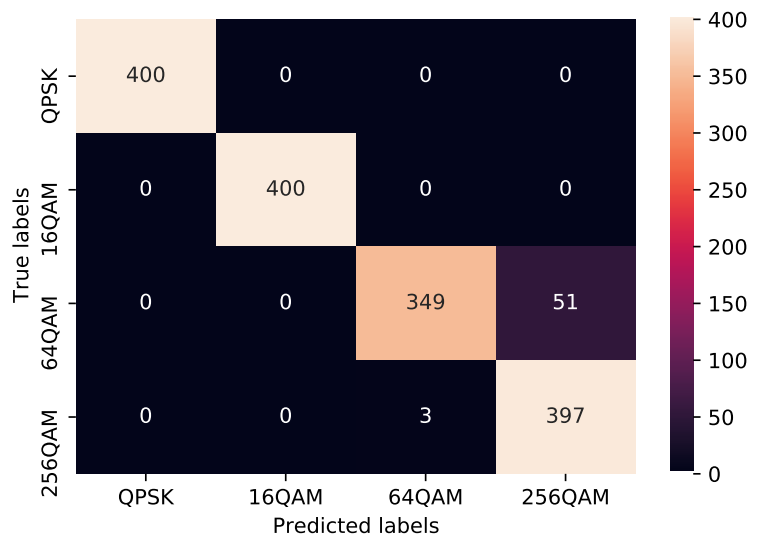

(d)

Fig. 8. Confusion matrices at 27.1-dB OSNR for (a) k-means, (b) fuzzy $c$-means, (c) PAPR and (d) KNN algorithms.

The matrices of the DNN and SVM methods were omitted since the methods achieved an accuracy equal to $100 \%$ and, therefore, the matrices are diagonal and do not add new information about their performance. In general, the results show that the performance of the other MFI algorithms is worse for high-order modulation formats. The classification errors are mostly caused by the imperfect blind pre-equalization carried out by the CMA, whose performance is worse for higher-order modulation formats. The k-means and fuzzy c-means are biased to selecting the QPSK for signals with a high number of constellation points with imperfect equalization and noise. The tendency of the accuracy curves in attaining a ceiling value indicates that they are mostly affected by the imperfect equalization and the small Euclidean distance between the constellation points. Moreover, increasing the OSNR even further will have a small effect on the average accuracy. For the PAPR, the highest number of classification errors occurs for 64QAM signals that are identified as 256QAM, indicating that the distribution of PAPR values for different realizations of the signal for a given modulation format may not be Gaussian or the variance may be dependant on the modulation format. This statement indicates 
that the decision threshold values could be optimized, requiring further investigation. However, this optimization does not affect the presented analysis as it is focused on the OSNR values in which the methods attain $100 \%$ average accuracy. The behavior is similar for the KNN algorithm.

\section{Conclusions}

A simulation-based comparative performance analysis for six blind modulation format identification (MFI) approaches were presented in the context of high capacity optical systems. The investigation was performed considering methods based on the k-nearest neighbors (KNN), k-means, fuzzy c-means, deep neural networks (DNN), support-vector machine (SVM), and peak-to-average power ratio (PAPR) algorithms. In all analyzed scenarios, 64-GBd optical channels were transmitted, with the transmitted bits sequence being coded following the QPSK, 16QAM, 64QAM, or 256QAM modulation formats.

For the first analyzed scenario, which considered optical signals degraded only by the insertion of amplified spontaneous emission (ASE) from optical amplifiers, the best-performing methods were the KNN, k-means, SVM, and PAPR, all requiring a received OSNR equal to $20.8 \mathrm{~dB}$ to achieve $100 \%$ accuracy on the modulation format identification. Still on the ASE-only scenario, the DNN reached $100 \%$ accuracy at $22.8 \mathrm{~dB}$ and the fuzzy c-means was not able to correctly identify 256QAM signals, therefore it never achieved values of average accuracy higher than $76 \%$. Following, the MFI algorithms performance was also characterized in a scenario considering signal degradation due to the ASE noise insertion, residual frequency deviation, and lasers linewidth. As the algorithms based solely on the analysis of the signal absolute amplitude information are not affected by phase and frequency deviations, only the k-nearest neighbors, $k$-means, and fuzzy c-means results were presented. The first two methods showed a 5-dB OSNR penalty to achieve the $100 \%$ accuracy, while the fuzzy c-mean algorithm attains $76 \%$ accuracy with a penalty of $4 \mathrm{~dB}$. Finally, for the more complex scenario, besides the ASE insertion and phase and frequency deviation effects, the degradation due to differential group delay (DGD) and polarization rotation was also considered. In this case, almost all algorithms were impacted by the constant modulus algorithm (CMA) non-optimal performance, especially for higherorder modulation formats, which was not able to compensate properly the degradation phenomena included in this scenario closer to a real implementation. The only two algorithms that were able to reach $100 \%$ accuracy in the range of received OSNRs simulated were the DNN and the SVM. It is important to highlight that, besides being trained employing only sequences with OSNRs equal to $26.4 \mathrm{~dB}$, the DNN was able to achieve high mean accuracy for all OSNRs employed during the validation stage. Nonetheless, the impact of training employing a wider range of OSNRs must be further investigated in future works. The DNN presented a penalty to the ASE-only scenario of $2.5 \mathrm{~dB}$, while the required received OSNR for the SVM to get 100\% accuracy is the same as in the ASE-only case, although the simulation of intermediate OSNR values are required to precisely assess the performance penalty. Comparing the complexity of both methods that achieved $100 \%$ accuracy, the SVM has fewer parameters to be adjusted than the DNN and is, therefore, easier and faster to be optimized, making it a good candidate for MFI. In addition, results for the most realistic simulation scenario show that the SVM solution achieved 100\% average accuracy at the lowest OSNR value and, consequently, could be used for longer transmission links without the use of regenerators.

Future works related to the results here presented will also include a more detailed computational complexity and latency comparison between the MFI approaches. The results of these investigations 
could present enough information to allow the definition of the best methods to perform the format modulation identification in next-generation optical transceivers.

\section{ACKNOWLEDGMENT}

This work was partially supported by FUNTTEL/Finep and CNPq.

\section{REFERENCES}

[1] Cisco. (2017) The Zettabyte Era: Trends and Analysis. [Online]. Available: https://www.cisco.com/c/en/us/solutions/ collateral/service-provider/visual-networking-index-vni/vni-hyperconnectivity-wp.html

[2] ITU-T Recommendation G.694. (2002) Spectral grids for WDM applications: DWDM frequency grid.

[3] O. Gerstel, M. Jinno, A. Lord, and S. J. B. Yoo, "Elastic optical networking: a new dawn for the optical layer?" IEEE Communications Magazine, vol. 50, no. 2, pp. s12-s20, February 2012, [doi:10.1109/MCOM.2012.6146481].

[4] R. Maher, A. Alvarado, D. Lavery, and P. Bayvel, "Modulation order and code rate optimisation for digital coherent transceivers using generalised mutual information," in Proc. of the European Conference on Optical Communication (ECOC'2015), pp. 1-3, Sept 2015, [doi:10.1109/ECOC.2015.7341621].

[5] P. Poggiolini, A. Carena, Y. Jiang, G. Bosco, and F. Forghieri, "On the ultimate potential of symbol-rate optimization for increasing system maximum reach,” in Proc. of the European Conference on Optical Communication (ECOC'2015), pp. 1-3, 2015, [doi:10.1109/ECOC.2015.7342025].

[6] Q. Zhang, H. Zhou, Y. Jiang, B. Cao, Y. Li, Y. Song, J. Chen, J. Zhang, and M. Wang, "A simple joint modulation format identification and OSNR monitoring scheme for IMDD OOFDM transceivers using K-nearest neighbor algorithm," Applied Sciences, vol. 9, no. 18, p. 3892, 2019, [doi:10.3390/app9183892].

[7] R. Boada, R. Borkowski, and I. T. Monroy, "Clustering algorithms for Stokes space modulation format recognition," Opt. Express, vol. 23, no. 12, pp. 15 521-15 531, Jun 2015, [doi:10.1364/OE.23.015521].

[8] F. N. Khan, K. Zhong, W. H. Al-Arashi, C. Yu, C. Lu, and A. P. T. Lau, "Modulation format identification in coherent receivers using deep machine learning," IEEE Photonics Technology Letters, vol. 28, no. 17, pp. 1886-1889, Sep. 2016, [doi:10.1109/LPT.2016.2574800].

[9] Z. Zhao, A. Yang, and P. Guo, "A modulation format identification method based on information entropy analysis of received optical communication signal," IEEE Access, vol. 7, pp. 41492-41497, 2019, [doi:10.1109/ACCESS.2019.2907521].

[10] S. M. Bilal, G. Bosco, Z. Dong, A. P. T. Lau, and C. Lu, "Blind modulation format identification for digital coherent receivers," Opt. Express, vol. 23, no. 20, pp. 26769-26778, Oct 2015, [doi:10.1364/OE.23.026769].

[11] A. L. N. Souza, T. Sutili, J. H. Cruz Júnior, and R. C. Figueiredo, "Avaliação de técnicas de inteligência artificial para identificação de formato de modulação em comunicações ópticas," in Anais do 19o Simpósio Brasileiro de Micro-ondas e Optoeletrônica and 14o Congresso Brasileiro de Eletromagnetismo (MOMAG'2020), pp. 51-55, 2020.

[12] B. J. Jain and K. Obermayer, "Elkan's k-means algorithm for graphs," in Advances in Soft Computing, pp. 22-32, 2010, [doi:10.1007/978-3-642-16773-7_2].

[13] P. J. Rousseeuw, "Silhouettes: A graphical aid to the interpretation and validation of cluster analysis," Journal of Computational and Applied Mathematics, vol. 20, pp. 53 - 65, 1987, [doi:10.1016/0377-0427(87)90125-7].

[14] "Selecting the number of clusters with Silhouette analysis on KMeans clustering." [Online]. Available: https://scikit-learn.org/stable/auto_examples/cluster/plot_kmeans_silhouette_analysis.html\# sphx-glr-auto-examples-cluster-plot-kmeans-silhouette-analysis-py

[15] T. J. Ross et al., Fuzzy Logic with Engineering Applications. Wiley Online Library, 2004, vol. 2.

[16] Z. Dong, K. Zhong, X. Zhou, C. Lu, A. P. T. Lau, Y. Lu, and L. Li, "Modulation-format-independent OSNR monitoring insensitive to cascaded filtering effects by low-cost coherent receptions and RF power measurements," Opt. Express, vol. 23, no. 12, pp. 15971-15982, Jun 2015, [doi:10.1364/OE.23.015971].

[17] M. Sokolova and G. Lapalme, "A systematic analysis of performance measures for classification tasks," Information Processing \& Management, vol. 45, no. 4, pp. 427-437, 2009, [doi:10.1016/j.ipm.2009.03.002].

[18] A. L. N. Souza, E. J. M. Ruiz, J. D. Reis, L. H. H. Carvalho, J. R. F. Oliveira, D. S. Arantes, M. H. M. Costa, and D. A. A. Mello, "Parameter selection in optical networks with variable-code-rate superchannels," J. Opt. Commun. Netw., vol. 8, no. 7, pp. A152-A161, Jul 2016, [doi:10.1364/JOCN.8.00A152].

[19] P. Poggiolini, “A generalized GN-model closed-form formula,” 2018. [Online]. Available: https://arxiv.org/ftp/arxiv/ papers/1810/1810.06545.pdf 
Journal of Microwaves, Optoelectronics and Electromagnetic Applications, Vol. 20, No. 4, December 2021 DOI: http://dx.doi.org/10.1590/2179-10742021v20i4254759

[20] D. Semrau, E. Sillekens, R. I. Killey, and P. Bayvel, "The ISRS GN model, an efficient tool in modeling ultra-wideband transmission in point-to-point and network scenarios," in 2018 European Conference on Optical Communication (ECOC), pp. 1-3, 2018, [doi:10.1109/ECOC.2018.8535146].

[21] D. Godard, "Self-recovering equalization and carrier tracking in two-dimensional data communication systems," IEEE T. Commun., vol. 28, no. 11, pp. 1867-1875, Nov 1980, [doi:10.1109/TCOM.1980.1094608]. 